\title{
Comparison between the compressibilities of the zero field and composite-fermion metallic states of the two-dimensional electron system
}

\author{
S. I. Dorozhkin, ${ }^{1}$ J. H. Smet,${ }^{2}$ K. von Klitzing, ${ }^{2}$ V. Umansky, ${ }^{3}$ R. J. Haug, ${ }^{4}$ and K. $\operatorname{Ploog}^{5}$ \\ ${ }^{1}$ Institute of Solid State Physics, Chernogolovka, Moscow district, 142432, Russia \\ ${ }^{2}$ Max-Planck-Institut für Festkörperforschung, Heisenbergstraße 1, D-70569 Stuttgart, Germany \\ ${ }^{3}$ Braun Center for Submicron Research, Weizmann Institute of Science, Rehovot 76100, Israel \\ ${ }^{4}$ Institut für Festkörperphysik, Universität Hannover, Appelstraße 2, 30167 Hannover, Germany \\ ${ }^{5}$ Paul-Drude Institut für Festkörperelektronik, Hausvogteiplatz 5-7, 10117 Berlin, Germany \\ (Received 24 August 2000; revised manuscript received 3 January 2001; published 5 March 2001)
}

\begin{abstract}
We have studied the capacitance between a two-dimensional electron system and a gate of field-effect transistors, produced from three different wafers with a single remotely doped $\mathrm{GaAs} / \mathrm{Al}_{x} \mathrm{Ga}_{1-x} \mathrm{As}_{\text {seterojunc- }}$ tion. In the vicinity of the Landau-level filling factor $\nu=1 / 2$, the increment of the capacitance relative to its zero-magnetic-field value, $\delta C_{1 / 2}$, was found to be insensitive to the carrier density, and close to the value as if the particles are noninteracting. This observation implies that electron-electron interaction affects the compressibility of the zero-field and composite-fermion metallic states in a very similar manner.
\end{abstract}

DOI: 10.1103/PhysRevB.63.121301

PACS number(s): 72.20.Fr, 72.20.My, 73.40.Kp

The composite-fermion picture ${ }^{1,2}$ (for a review, see Refs. 3 and 4) provides a unified approach for describing the properties of a two-dimensional electron system (2DES) in the integer and fractional quantum Hall regimes. At spin-split Landau-level filling factors $\nu=1 / 2 n$ (where $n$ is an integer), it predicts the existence of metallic states composed of composite quasiparticles, each made up of one electron and $2 n$ flux quanta. At exact $1 / 2 n$ filling, these composite fermions (CF's) experience a zero effective magnetic field. Departure from these filling factors results in the appearance of a nonzero effective magnetic field, and leads to the discretization of the composite-fermion energy spectrum due to Landau quantization. Within this approach, the fractional quantum Hall effect of conventional electrons is mapped onto the integer quantum Hall effect of CF's. Substantial experimental evidence that this model captures the essential physics in the vicinity of $\nu=1 / 2 n$ filling comes from commensurability and surface acoustic wave experiments. ${ }^{5}$ For these experiments the basic assumption of the composite-fermion theory, that dressing the electrons with $2 n$-flux quanta constitutes the main effect of the Coulomb interaction between the 2D electrons while any remaining interaction may be ignored, appears well justified. Even though residual interaction exists, these results are insensitive to it because they rely on a geometrical comparison between an externally imposed length scale and the Fermi-wavelength-related distance, the cyclotron radius, a quantity that depends solely on the carrier density. ${ }^{2}$ Because of the Landau-level degeneracy the energy dispersion of CF's is not described by the band mass of the host semiconductor material, but by an "effective mass", originating from the Coulomb energy. Various methods, such as temperature-dependent dc transport, ${ }^{6}$ thermopower, ${ }^{7}$ and photoluminescence techniques, ${ }^{8}$ were used to probe the effective mass on approaching $\nu=1 / 2 n$. But considerable controversy remains.

Here we present results directly related to the compressibility,

$$
\kappa=\frac{1}{n_{s}^{2}}\left(\frac{d \mu}{d n_{s}}\right)^{-1},
$$

at filling factors $\nu=1 / 2 n$, with $n_{s}$ the areal density of electrons and $\mu$ the chemical potential calculated from the bottom of the ground subband. This thermodynamic quantity contains not only a contribution due to the finite density of states at the Fermi energy, determined by the CF effective mass, but also exchange/correlation terms. Up to now, published data at $\nu=1 / 2$ were limited to a few isolated points. ${ }^{9-11}$ We performed a systematic study as a function of electron density $n_{s}$ in a range from $6 \times 10^{10}$ to $18 \times 10^{10}$ $\mathrm{cm}^{-2}$, and derived the following conclusion: The contributions of the interaction terms to the inverse compressibility of the zero-field electron and composite-fermion metallic states at a given areal density are nearly identical, since the difference of the derivatives $d \mu / d n_{s}$, measured at zero field and at $\nu=1 / 2$ or $1 / 4$, does not depend on $n_{s}$, and is close to the value expected for noninteracting electrons.

To characterize the compressibility, we have measured the capacitance between the 2DES and the gate of a fieldeffect transistor, $C \equiv d Q / d V_{g}$, where $Q$ is the charge on the capacitor and $V_{g}$ is the gate voltage. The inverse value of the capacitance includes a term proportional to the derivative $d \mu / d n_{s}$. It reads (see, for example, Ref. 12, and references therein)

$$
\frac{S}{C}=\frac{S}{C_{g}}+\frac{S}{C_{z}}+\frac{1}{e^{2}} \frac{d \mu}{d n_{s}},
$$

where $S$ is the gated area of the sample. The largest term in this equation usually originates from the geometrical capacitance, $C_{g}=\epsilon_{i} S / d_{0}$, where $d_{0}$ is the thickness of the insulating layer, with permittivity $\epsilon_{i}$ separating the gate and the GaAs/ $\mathrm{Al}_{x} \mathrm{Ga}_{1-x} \mathrm{As}$ interface. The second term in Eq. (2) is a correction to the geometrical capacitance due to the finite width of the 2DES and the band profile modifications induced by the dopants. The quantity $C_{\mathrm{z}}$ can be written in the form $C_{z}=\epsilon S / z_{0}$, where $\epsilon$ is the permittivity of GaAs and $z_{0}\left(n_{s}, n_{i}\right)$ is a smooth function of the electron density of the 2DES, $n_{s}$, and the areal density of charged impurities, $n_{i}$. It 
TABLE I. Sample parameters: $S$ is the gated area, $d_{0}$ is the distance from the $\mathrm{GaAs} / \mathrm{Al}_{x} \mathrm{Ga}_{1-x} \mathrm{As}$ heterointerface to the wafer surface (as grown), $\delta$ is the width of the spacer (as grown), $n_{s}\left(V_{g}\right.$ $=0)$ and $\mu_{0}\left(V_{g}=0\right)$ are the areal electron density and the mobility at zero gate voltage, respectively.

\begin{tabular}{lccccc}
\hline \hline \multicolumn{1}{c}{ Sample } & $\begin{array}{c}S \\
\mathrm{~mm}^{2}\end{array}$ & $\begin{array}{c}d_{0} \\
\AA\end{array}$ & $\begin{array}{c}\delta \\
\AA\end{array}$ & $\begin{array}{c}n_{s}\left(V_{g}=0\right) \\
\mathrm{cm}^{-2}\end{array}$ & $\begin{array}{c}\mu_{0}\left(V_{g}=0\right) \\
\mathrm{cm}^{2} / \mathrm{Vs}\end{array}$ \\
\hline 1 before illum. & 5.06 & 1100 & 850 & $1.0 \times 10^{11}$ & $1.3 \times 10^{6}$ \\
1 after illum. & 5.06 & 1100 & 850 & $1.7 \times 10^{11}$ & $1.5 \times 10^{6}$ \\
2 & 0.82 & 1100 & 400 & $1.8 \times 10^{11}$ & $1.1 \times 10^{6}$ \\
3 & 0.92 & 5800 & 700 & $1.4 \times 10^{11}$ & $1.2 \times 10^{6}$ \\
\hline \hline
\end{tabular}

depends on the sample architecture, and has typical values of $z_{0} \sim 100 \AA$ ( $\approx$ the width of the wave function in the confining potential). The third term describes the thermodynamic properties of the 2DES, and is normally of the same order as the second term. Equation (2) is valid if the following conditions are satisfied: (i) A variation of the gate voltage induces a change of the charge in the 2DES and on the gate only. Impurity charges must remain unaffected (frozen). The validity of this assumption for our samples is verified below. (ii) The size quantization can be treated separately from the electron motion within the plane. This is generally accepted for the ground subband of $2 \mathrm{D}$ electron systems. The second term of Eq. (2), then, is not influenced by the perpendicular magnetic field and the Landau quantization. Consequently, the difference of the inverse capacitance measured at $B(\nu$ $=1 / 2 n$ ) and $B=0$ contains only those terms describing the thermodynamical characteristics of the 2DES in these two states:

$$
\begin{aligned}
\delta\left(\frac{1}{C}\right)_{1 / 2 n} & =\left.\frac{1}{C}\right|_{\nu=1 / 2 n}-\left.\frac{1}{C}\right|_{B=0} \\
& =\frac{1}{e^{2} S}\left(\left.\frac{d \mu}{d n_{s}}\right|_{\nu=1 / 2 n}-\left.\frac{d \mu}{d n_{s}}\right|_{B=0}\right) .
\end{aligned}
$$

The magnetic-field-induced increment in the capacitance at $\nu=1 / 2 n, \delta C_{1 / 2 n}=\left.C\right|_{\nu=1 / 2 n}-\left.C\right|_{B=0}$, can thus be expressed as

$$
\delta C_{1 / 2 n}=\frac{\left.\left.C\right|_{\nu=1 / 2 n} C\right|_{B=0}}{e^{2} S}\left(\left.\frac{d \mu}{d n_{s}}\right|_{B=0}-\left.\frac{d \mu}{d n_{s}}\right|_{\nu=1 / 2 n}\right) .
$$

For our experiments we used field-effect transistors with Schottky gates, processed on three different wafers. The parameters are summarized in Table I. The wafers have the standard single remotely-doped $\mathrm{Al}_{x} \mathrm{Ga}_{1-x} \mathrm{As} / \mathrm{GaAs}$ heterojunction architecture with either volume doping (sample 1 and 2) or a $\delta$-doped layer (sample 3 ). Note that the distance $d_{0}$ between the gate and the heterointerface varies from sample to sample by about a factor 5.5. Sample 1 was measured before and after illumination to modify the electron areal density at zero gate bias. To measure capacitance an ac voltage was applied between the $2 \mathrm{DES}$ and the gate via coaxial cables, and the ac-current response was detected with

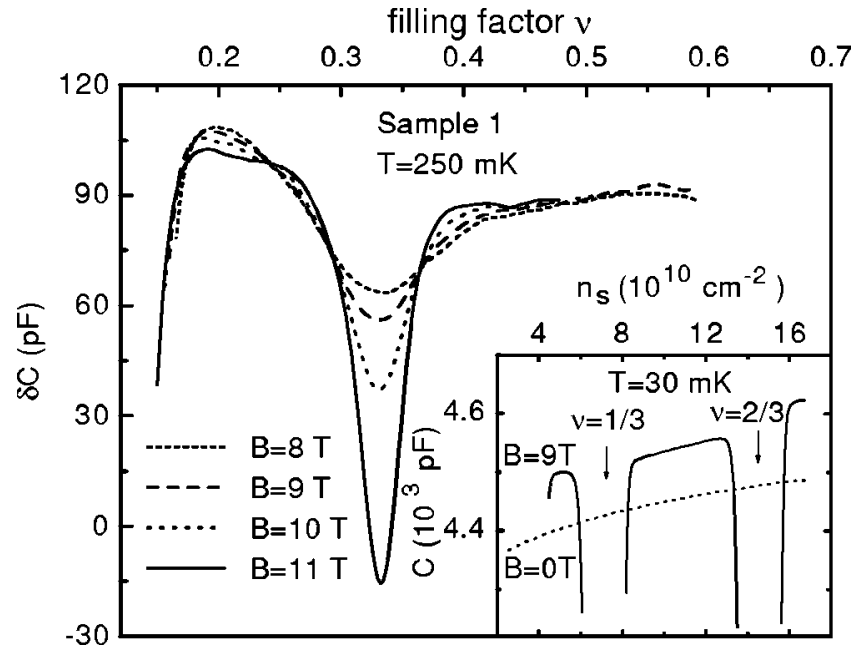

FIG. 1. The difference in capacitance at $B=8,9,10$, and $11 \mathrm{~T}$, and at zero magnetic field $\delta C\left(B, n_{s}\right)=C\left(B, n_{s}\right)-C\left(B=0, n_{s}\right)$, vs filling factor $\nu=n_{s} /(e B / h)$ for sample 1 prior to illumination. The inset shows the capacitance $C$ as a function of the electron areal density $n_{s}$ measured at $B=0$ and $B=9$ T for sample 1 after illumination.

the aid of a current-to-voltage converter and a lock-in amplifier. The circuitry minimizes parasitic capacitance contributions, and allows one to determine the correct phase shift between the modulation voltage and the current. The excitation voltage with frequency $9.2 \mathrm{~Hz}$ was equal to $4 \mathrm{mV}$ for samples 1 and 2, and $15 \mathrm{mV}$ for sample 3. It was verified that the modulation was sufficiently low to ensure no influence on the shape of the magnetocapacitance curves even at the fractional quantum Hall minima (see Figs. 1 and 2). The main part (about 95\%) of the signal from the current-tovoltage converter was compensated for by the signal from the same oscillator, that provided the excitation voltage, but appropriately adjusted in amplitude and phase. This essentially diminishes the influence of oscillator drift in the amplitude and frequency. As a result, we were able to resolve variations in the capacitance of about $2 \times 10^{-4}$. After calibration of the current-to-voltage converter and lock-in ampli-

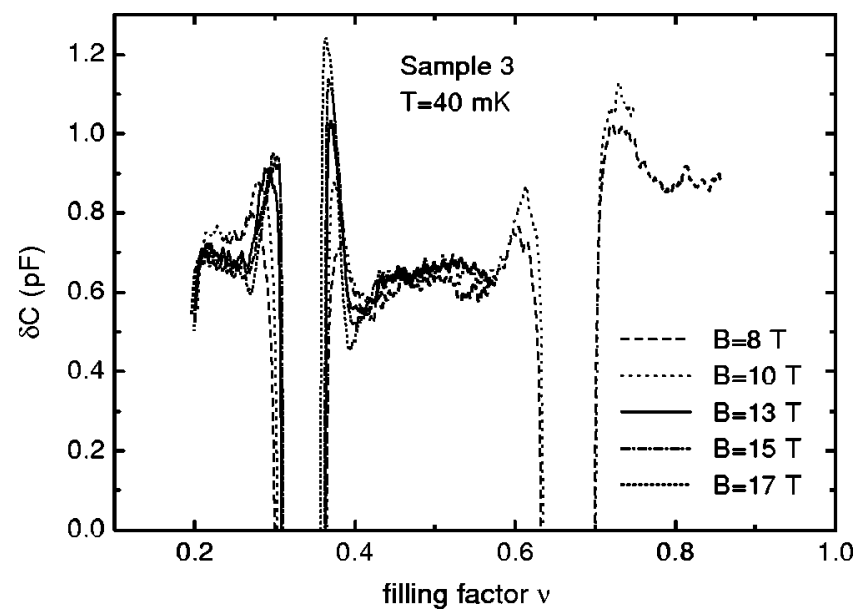

FIG. 2. The same as in Fig. 1 for sample 3. 


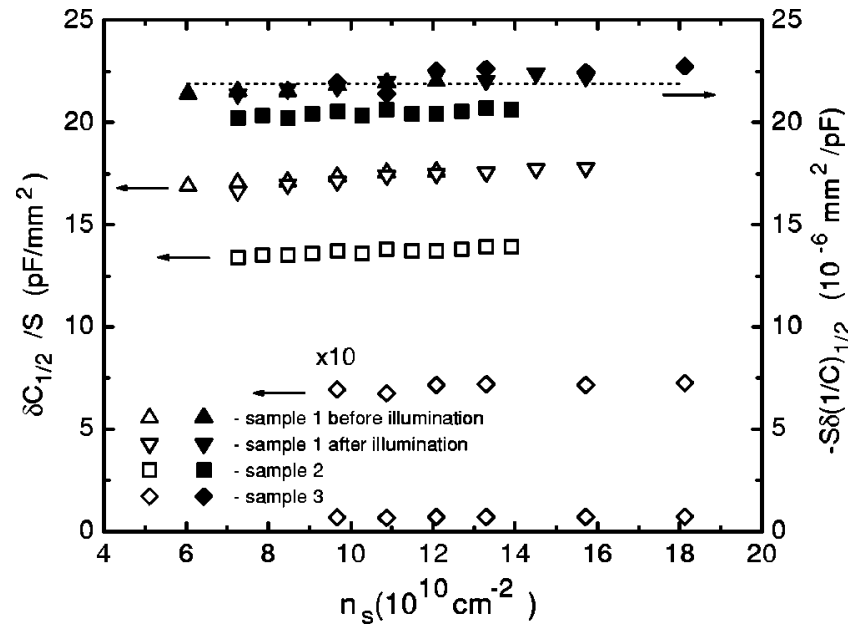

FIG. 3. Experimental values of $\delta C_{1 / 2} / S$ (open symbols) and $-S \delta(1 / C)_{1 / 2}=S \delta C_{1 / 2} /\left(\left.\left.C\right|_{\nu=1 / 2} C\right|_{B=0}\right.$ ) (closed symbols) vs electron areal density for all three samples investigated. The dashed line represents the value of $\pi \hbar^{2} /\left(m * e^{2}\right)$, which is expected for $-S \delta(1 / C)_{1 / 2}$ in the case of noninteracting $2 \mathrm{D}$ electrons with $m^{*}$ $=0.068 m_{e}$ and unbroadened Landau levels.

fier, an accuracy in the capacitance values of better than $1 \%$ was achieved. The same accuracy holds for the capacitance difference $\delta C_{1 / 2}$ for samples 1 and 2. For sample 3 this difference is substantially smaller, and the accuracy is limited by the noise level to approximately $4 \%$. The absolute values of the measured capacitances agree within $2 \%$ for samples 2 and 3 , and $5 \%$ for sample 1 , with the calculated values deduced from the period of the Shubnikov-de Haas oscillations as a function of the gate voltage. The uncertainty arises mainly from the uncertainty in the area of the transistor. This supports our assumption of no additional charge reservoirs in the derivation of Eq. (2). ${ }^{13}$

An example of raw experimental capacitance data as functions of the areal electron density is depicted in the inset to Fig. 1. The deep minima at fractional filling factors $1 / 3$ and $2 / 3$ are mainly due to resistive effects caused by the small values of the longitudinal conductivity $\sigma_{x x}$ in this regime. In the absence of such effects at higher temperature, the minima become less pronounced, and reflect the chemical potential discontinuity at corresponding fractional fillings (see, for example, Ref. 14). Away from the fractional quantum Hall effect states (particularly, in the vicinity of $\nu$ $=1 / 2$ and $1 / 4$ ), the resistive effects are absent down to the lowest temperatures. In the main part of this figure the difference $\delta C\left(B, n_{s}\right)=C\left(B, n_{s}\right)-C\left(B=0, n_{s}\right)$, between the curves measured in the presence of a constant magnetic field $B$ and those measured at zero magnetic field, are presented for various $B$ values. The data are plotted with the filling factor $\nu$ as abscissa. At fractional quantum Hall effect states, $\delta C$ depends on the magnetic field, but close to filling factors $1 / 2$ and $1 / 4$ it approaches the same value for measurements at different magnetic fields. This means that $\delta C_{1 / 2}$ and $\delta C_{1 / 4}$ do not depend on the carrier density $n_{s}$. This behavior was established for all samples. Data acquired on sample 3 are illustrated in Fig. 2, and a summary of the data at $\nu=1 / 2$ for all samples is shown in Fig. 3. Note that capacitance values at $B=0$ and $\nu=1 / 2$ and $1 / 4$ were found to be temperature independent up to at least $T=2 \mathrm{~K}$. According to Eq. (3) when considering the quantity $S \delta(1 / C)_{1 / 2 n}$ the, for this study irrelevant, geometric capacitance contribution and its correction due to the finite width of the $2 \mathrm{D}$ channel conveniently drop out, leaving nothing but a term proportional to the difference of the inverse compressibilities. This is also included in Fig. 3 for filling factor $\nu=1 / 2$ (solid symbols). Even though the absolute values of $\delta C_{1 / 2} / S$ for the samples listed in Table I differ by more than a factor of 20 due to their very different $d_{0}$ values and gated area, as illustrated in Fig. 3, $-S \delta(1 / C)_{1 / 2}$ is nearly the same for all samples, and is, moreover, independent of the carrier density. It takes on the same value that one would obtain if one ignored Landaulevel broadening and interaction among $2 \mathrm{D}$ electrons with an effective mass of $m^{*}=0.068 m_{e}$ for which $d \mu /\left.d n_{s}\right|_{B=0}$ $\rightarrow \pi \hbar^{2} / m^{*}$ and $d \mu /\left.d n_{s}\right|_{\nu=1 / 2} \rightarrow 0$ :

$$
-S \delta\left(\frac{1}{C}\right)_{1 / 2}=\frac{\pi \hbar^{2}}{m^{*} e^{2}} .
$$

A similar fact holds, albeit with poorer accuracy (about $15 \%$ ), for $\nu=1 / 4$. If indeed interaction contributions to the compressibility of the 2DES at zero magnetic field and halffilling are ignored, then this result would merely confirm the large density-of-states mass at half-filling previously reported on the basis of temperature-dependent dc-transport data $^{6}$ and photoluminescence measurements ${ }^{8}$ at exact halffilling. However, it is generally accepted that under usual experimental conditions the compressibility of the 2DES is strongly affected by the electron-electron interaction. In particular, it was found ${ }^{10,11,15,16}$ that at zero magnetic field $d \mu / d n_{s}$ becomes negative at $n_{s}<1 \times 10^{11} \mathrm{~cm}^{-2}$. Moreover, a theoretical formula, ${ }^{17}$ that describes the electron-electron interaction contribution within a Hartree-Fock approximation, provides a reasonable estimate for the measured quantity:

$$
\left.\frac{d \mu}{d n_{s}}\right|_{B=0}=\frac{\pi \hbar^{2}}{m^{*}}-\left(\frac{2}{\pi}\right)^{1 / 2} \frac{e^{2}}{4 \pi \epsilon \sqrt{n_{s}}} .
$$

Agreement with the experiment becomes much better if the softening of the Coulomb interaction due to finite thickness of the 2DES is taken into account. ${ }^{11}$ The second, densitydependent term in Eq. (6), absent in the case of noninteracting $2 \mathrm{D}$ electrons, varies by almost a factor of 1.6 for the density range covered by the experiment, and cancels the first term (single particle contribution) at a density of 6.5 $\times 10^{10} \mathrm{~cm}^{-2}$. It is therefore well within our experimental resolution.

We have also examined the experimental data published by other authors. Those results ${ }^{9-11}$ that allow a determination of the difference $d \mu /\left.d n_{s}\right|_{B=0}-d \mu /\left.d n_{s}\right|_{\nu=1 / 2}$ from the raw experimental curves agree well with our findings (in Ref. 11 a few data points for electron densities that differ by up to a factor of 2 are presented). Note, that in Refs. 10 and 11 an alternative experimental technique was utilized, that enables one to measure absolute values of the electron compressibility. 
These results for the difference in $d \mu / d n_{s}$ imply that the electron-electron interaction contributions to the inverse compressibility of the zero field and composite-fermion metallic states are nearly identical. This is a remarkable coincidence and has, to the best of our knowledge, not been reported experimentally or predicted theoretically. The phenomenological interpolation formula ${ }^{18}$ for the groundstate energy of a two-dimensional electron system in the extreme quantum limit describes only a partial cancellation of interaction-induced terms in the inverse compressibilities. It yields, in combination with Eq. (6),

$$
\left.\frac{d \mu}{d n_{s}}\right|_{B=0}-\left.\frac{d \mu}{d n_{s}}\right|_{\nu=1 / 2}=\frac{\pi \hbar^{2}}{m^{*}}+\frac{e^{2}}{4 \pi \epsilon \sqrt{n_{s}}}(\sqrt{\pi} K-\sqrt{2 / \pi}) .
$$

The first term in the brackets cancels at best up to $76 \%$ of the second term for the values of coefficient $K$, obtained from parameters that enter this interpolation formula as described in Ref. 18. While the softening of the Coulomb interaction in real quasi-two-dimensional systems might, in some circumstances, be one of the mechanisms leading to a more complete cancellation of the inverse compressibilities than given by Eq. (7), the insensitivity of our results on the electron density and the particular sample geometry, that both strongly influence the softening, points to a more fundamental cause.

In conclusion, we find that density-dependent electronelectron interaction contributions cancel when considering the difference of the inverse compressibility of the zero-field and composite-fermion metallic states of the twodimensional electron system. In view of the density range investigated in this work, as well as the very different samples measured, the cancellation is unlikely to be fortuitous and may be of fundamental origin.

Partial support of this work by INTAS and RFBR (S.I.D.), and the Deutsche Forschungsgemeinschaft (J.H.S.) is gratefully acknowledged.
${ }^{1}$ J. K. Jain, Phys. Rev. Lett. 63, 199 (1989).

${ }^{2}$ B. I. Halperin, P. A. Lee, and N. Read, Phys. Rev. B 47, 7312 (1993).

3 B. I. Halperin, in Perspectives in Quantum Hall Effects, edited by S. Das Sarma and A. Pinczuk (Wiley, New York, 1996).

${ }^{4}$ J. K. Jain, in Perspectives in Quantum Hall Effects (Ref. 3).

${ }^{5}$ See, for example, R. L. Willett et al., Phys. Rev. Lett. 71, 3846 (1993); W. Kang et al., ibid. 71, 3850 (1993); J. H. Smet et al., ibid. 83, 2620 (1999).

${ }^{6}$ See, for a review, H. L. Stormer and D. C. Tsui, in Perspectives in Quantum Hall Effects (Ref. 3); R. L. Willett, Semicond. Sci. Technol. 12, 495 (1997).

${ }^{7}$ B. Tieke et al., Phys. Rev. Lett. 76, 3630 (1996).

${ }^{8}$ I. V. Kukushkin, K. von Klitzing, and K. Eberl, Phys. Rev. Lett. 82, 3665 (1999).

${ }^{9}$ T. P. Smith III, W. I. Wang, and P. J. Stiles, Phys. Rev. B 34, 2995 (1986).

${ }^{10}$ J. P. Eisenstein, L. N. Pfeiffer, and K. W. West, Phys. Rev. Lett.
68, 674 (1992).

${ }^{11}$ J. P. Eisenstein, L. N. Pfeiffer, and K. W. West, Phys. Rev. B 50, 1760 (1994).

${ }^{12}$ T. P. Smith, B. B. Goldberg, P. J. Stiles, and M. Heiblum, Phys. Rev. B 32, 2696 (1985).

${ }^{13}$ Our calculations show that the uncertainty, introduced by the possible presence of unfrozen impurities in determining $d \mu /\left.d n_{s}\right|_{B=0}-d \mu /\left.d n_{s}\right|_{\nu=1 / 2}$ using our method, does not exceed $2\left|S e d n_{s} / d V_{g}-C\right| / C$ (4\% for samples 2 and 3, and $10 \%$ for sample 1), where the quantity $d n_{s} / d V_{g}$ is obtained from the Shubnikov-de Haas oscillations.

${ }^{14}$ S. I. Dorozhkin et al., Phys. Rev. B 51, 14729 (1995).

${ }^{15}$ S. Shapira et al., Phys. Rev. Lett. 77, 3181 (1996).

${ }^{16}$ S. Ilani et al., Phys. Rev. Lett. 84, 3133 (2000).

${ }^{17}$ S. Nagano, K. S. Singwi, and S. Ohnishi, Phys. Rev. B 29, 1209 (1984).

${ }^{18}$ G. Fano and F. Ortolani, Phys. Rev. B 37, 8179 (1988). 